\title{
Ausgezeichnete Innovatoren
}

\author{
Zum 30. Mal wurde der Galenus-von-Pergamon-Preis verliehen, und zwar \\ in den vier Kategorien Grundlagenforschung, Primary Care, Specialist Care \\ und Orphan Drugs. Außerdem gab es in Berlin den CharityAward für \\ ehrenamtliches Engagement in der Gesundheitsversorgung.
}

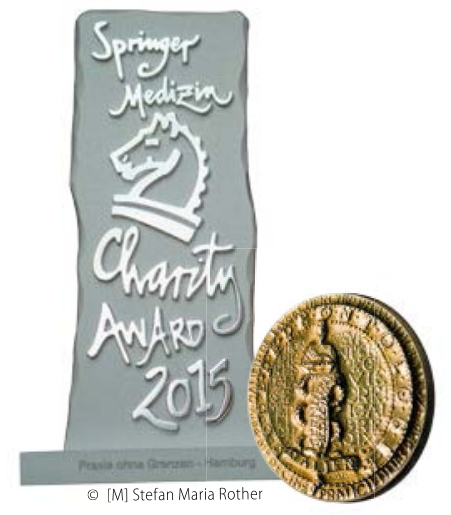

Die Preisträger des Galenus-von-Pergamon-Preises und des CharityAwards 2015 stehen fest: Am 15. Oktober 2015 wurden die von Springer Medizin gestifteten Preise in Berlin verliehen. Über 200 geladene Gäste kamen im Axica am Brandenburger Tor zusammen, um hautnah mitzuerleben, welche herausragenden Leistungen in der Arzneimittelforschung und in ehrenamtlichem Engagement ausgezeichnet werden. Beides, pharmazeutischer Fortschritt und Ehrenamt, sind unverzichtbare Leistungen, die zu einer besseren Gesellschaft beitragen. Auch in diesem Jahr wurden Galenus-Preise in vier Kategorien vergeben:

- pharmakologische Grundlagenforschung,

- Primary Care,

- Specialist Care und

- Orphan Drug.

\section{Impulse für den Fortschritt}

Den Galenus-von-Pergamon-Preis in der Kategorie beste pharmakologische Grundlagenforschung nahm in diesem Jahr Prof. Jan Eric Siemens vom Pharmakologischen Institut der Universität Heidelberg entgegen. Er und sein Team erforschten, wie man mittels Botenstoffen Nervenzellen weniger reizbar machen kann. Sie fanden heraus, dass man über die Aktivierung des $\mathrm{GABA}_{\mathrm{B1}}$-Rezeptors eine Sensibilisierung des Schmerzrezeptor sTRPV1 verhindern oder aufheben kann.
In der Kategorie Primary Care wurde Nalmefen (Selincro $\left.{ }^{\circ}\right)$ von Lundbeck mit dem Galenus-Preis ausgezeichnet. Hierbei handelt es sich um einen Opioidsystem-Modulator, mit dem sich der Alkoholkonsum bei erwachsenen Patienten mit Alkoholabhängigkeit vermindern lässt. Im Bereich Specialist Care erhielt Bayer HealthCare mit Xofigo ${ }^{\circ}$ (Radium-223-dichlorid) den Preis. Xofigo ${ }^{\circ}$ wird zur Therapie von Knochenmetastasen bei Prostatakrebs eingesetzt. Durch den Alpha-Strahler können gezielt Tumorzellen abgetötet werden, wobei das umgebende Gewebe und das Knochenmark nur einer vergleichsweise geringen Strahlenbelastung ausgesetzt sind. Das Gesamtüberleben der Patienten und die Zeit bis zum Auftreten von symptomatischen skelettalen Ereignissen können so deutlich verlängert werden.

Seit dem Jahr 2014 werden auch Arzneimittelinnovationen im Bereich Orphan Drugs für Patienten mit seltenen Erkrankungen geehrt. In dieser Kategorie wurde in diesem Jahr Translarna (Ataluren) von PTC Therapeutics ausgezeichnet. Dabei handelt es sich um die erste zugelassene Therapie mit kausaler Wirkung bei Duchenne-Muskeldystrophie mit zugrundeliegender Nonsense-Mutation. Das Medikament greift in die gestörte Bildung eines lebensnotwendigen Muskelproteins ein. Über alle vier Preise entschied wenige Stunden vor der Verleihung eine 14-köpfige Jury um Prof. Erland Erdmann, Köln, als Vorsitzendem.

\begin{abstract}
Ausgezeichnetes Engagement
Seit sechs Jahren ist mit der Auszeichnung der Preisträger des Galenus-vonPergamon-Preises die Würdigung bürgerschaftlichen Engagements durch den von Springer Medizin gestifteten CharityAward verbunden. „Kranke, pflege- und hilfsbedürftige Menschen brauchen mehr als Innovationen und Rechtsansprüche auf professionelle Versorgung. Sie brauchen auch die "Caring Community", sagte Bundesgesundheitsminister Hermann Gröhe, der Schirmherr des CharityAwards, bei der Preisverleihung im Oktober.

Platz 1 gewann „Praxis ohne Grenzen“. Gestartet war die Praxis mit 20 Ärzten und acht Krankenschwestern, inzwischen arbeiten 34 Ärzte, zwölf Krankenschwestern und zwei Dolmetscher in der "Praxis ohne Grenzen“ in Hamburg-Horn. Ehrenamtlich versorgen sie Menschen ohne Krankenversicherung - zwischen 50 und 60 Patienten je Sprechstunde, so der 1. Vorsitzende Prof. Peter C. Ostendorf.

Den zweiten Platz belegte „SeeYou - Babylotse“. Seit Februar 2004 bietet das Hamburger Kinderkrankenhaus Wilhelmstift familienorientierte Nachsorge an. Unter anderem mit dem ausgezeichneten Programm „Babylotse“ unterstützt die Stiftung werdende und frisch gebackene Eltern nach der Entlassung aus dem Krankenhaus. Dr. Sönke Siefert und Anna Kupfer von "SeeYou“ betonen: „Wir brauchen vor allem Öffentlichkeitsarbeit, um mehr Frauen- und Kinderärzte zu erreichen."
\end{abstract}

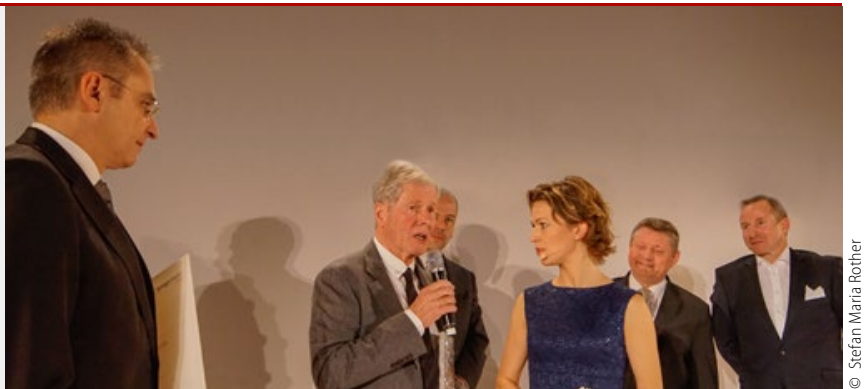

Peter C. Ostendorf (2. v. l.) freut sich über den ersten Platz beim CharityAward. Mit im Bild Joachim Krieger (l.), Springer Medizin, Markus Spamer (3.v.l.) und Tom Schweitzer (r.) von TV Wartezimmer, Moderatorin Anna Planken und Minister Gröhe.

Platz 3 erreichten „die LandArztMacher“. Die Initiative begleitet Medizinstudenten und bietet innen die Möglichkeit, in Landarztpraxen Erfahrung als angehende Hausärzte zu sammeln. „Wir meinen, es ist der schönste Beruf. Es macht unglaublich viel Spaß, Netzwerke mit Ärzten und anderen Berufen zu bilden", sagte Dr. Wolfgang Blank aus Kirchberg. Helmut Laschet 